\title{
Reduced Microvascular Thrombosis and Improved Outcome in Acute Murine Stroke by Inhibiting GP Ilb/llla Receptor-mediated Platelet Aggregation
}

\author{
Tanvir F. Choudhri, ${ }^{\star}$ Brian L. Hoh, ${ }^{*}$ Hans-Gunter Zerwes, ${ }^{\ddagger}$ Charles J. Prestigiacomo, ${ }^{\star}$ Samuel C. Kim, ${ }^{*}$ \\ E. Sander Connolly, Jr., ${ }^{\star}$ Georg Kottirsch, ${ }^{\ddagger}$ and David J. Pinsky $\$$ \\ College of Physicians and Surgeons, *Department of Neurological Surgery, ${ }^{\S}$ Department of Medicine, Columbia University, New York, \\ New York 10032; and ${ }^{\ddagger}$ Novartis Pharmaceuticals, Ch 4002 Basel, Switzerland
}

\begin{abstract}
Treatment options in acute stroke are limited by a dearth of safe and effective regimens for recanalization of an occluded cerebrovascular tributary, as well as by the fact that patients present only after the occlusive event is established. We hypothesized that even if the site of major arterial occlusion is recanalized after stroke, microvascular thrombosis continues to occur at distal sites, reducing postischemic flow and contributing to ongoing neuronal death. To test this hypothesis, and to show that microvascular thrombosis occurs as an ongoing, dynamic process after the onset of stroke, we tested the effects of a potent antiplatelet agent given both before and after the onset of middle cerebral arterial (MCA) occlusion in a murine model of stroke. After $45 \mathrm{~min}$ of MCA occlusion and $23 \mathrm{~h}$ of reperfusion, fibrin accumulates in the ipsilateral cerebral hemisphere, based upon immunoblotting, and localizes to microvascular lumena, based upon immunostaining. In concordance with these data, there is a nearly threefold increase in the ipsilateral accumulation of ${ }^{111}$ In-labeled platelets in mice subjected to stroke compared with mice not subjected to stroke. When a novel inhibitor of the glycoprotein IIb/IIIa receptor (SDZ GPI 562) was administered immediately before MCA occlusion, platelet accumulation was reduced $48 \%$, and fibrin accumulation was reduced by $47 \%$ by immunoblot densitometry. GPI 562 exhibited a dose-dependent reduction of cerebral infarct volumes measured by triphenyltetrazolium chloride staining, as well as improvement in postischemic cerebral blood flow, measured by laser doppler. GPI 562 caused a dose-dependent increase in tail vein bleeding time, but intracerebral hemorrhage (ICH) was not significantly increased at therapeutic doses; however, there was an increase in ICH at the highest doses tested. When given immediately after withdrawal of the MCA occluding suture, GPI 562 was shown to reduce cerebral infarct volumes by $70 \%$. These data support the hypothesis that in ischemic regions of brain, microvascular thrombi continue to accumu-
\end{abstract}

Address correspondence to David J. Pinsky, Columbia University, College of Physicians and Surgeons, PH 10 Stem, 630 W. 168th St., New York, NY 10032. Phone: 212-305-6071; FAX: 212-305-7638; E-mail: djp5@columbia.edu

Received for publication 6 March 1998 and accepted in revised form 11 August 1998.

J. Clin. Invest.

(C) The American Society for Clinical Investigation, Inc. 0021-9738/98/10/1301/10 \$2.00

Volume 102, Number 7, October 1998, 1301-1310

http://www.jci.org late even after recanalization of the MCA, contributing to postischemic hypoperfusion and ongoing neuronal damage. (J. Clin. Invest. 1998. 102:1301-1310.) Key words: platelets • stroke • fibrin • GP IIb/IIIa • thrombosis

\section{Introduction}

Acute ischemic stroke is usually caused by a cardio- or atheroembolic event, which occludes a major vascular tributary to the brain. Although fragments of lipid-laden, platelet-, and fibrin-rich debris can also migrate downstream beyond the site of the original occlusion, it is possible that in situ microvascular thrombosis may be triggered as well at these downstream sites, perhaps accounting for the observed accumulation of thrombotic material in branches of the microcirculation in a primate model of stroke (1-3). Additional evidence also supports this possibility, in that, in a murine model of stroke induced by temporarily obstructing a major vascular tributary with a nylon suture that is subsequently removed, cerebral blood flow $(\mathrm{CBF})^{1}$ does not return to preischemic levels after removal of the obstructing suture (4-6). Although neutrophilcapillary plugging is an important contributor to postischemic cerebrovascular no reflow, preischemic depletion of neutrophils (5) or blockade of specific neutrophil adhesion receptors (6) does not completely prevent postischemic no-reflow. Other mechanisms of postischemic no-reflow are, therefore, likely to be involved, perhaps related to in situ formation of microvascular thrombus at downstream sites. This might occur due to neutrophil-platelet interactions (7), or hypoxia-induced modulation of the vascular wall phenotype, rendering it highly prothrombotic $(8,9)$, with reperfusion being a potent stimulus for further platelet aggregation (10).

It is important to consider that microthrombi may actually contribute to infarct progression, given their topographic localization to microvessels distributed throughout the ischemic territory (11). In this regard, platelets are likely to be pathophysiological mediators of progressive tissue injury in stroke, accelerating the local accretion of thrombus in the cerebral microvasculature. In humans, patients with essential thrombocythemia have a propensity towards ischemic stroke (12). In animal models of ischemic stroke, scintigraphic studies in rats (13), cats $(14)$, and dogs $(15,16)$ show that ${ }^{111}$ Indium (In)labeled platelets accumulate in the ipsilateral ischemic cerebral cortex. Although the platelet inhibitor aspirin is effective in terms of primary prevention of stroke (17), it is not clear that aspirin is efficacious when used to treat acute stroke in evolution. Similarly, PGI2, which inhibits platelet aggregation

1. Abbreviations used in this paper: $\mathrm{CBF}$, cerebral blood flow; $\mathrm{ICH}$, intracerebral hemorrhage; MCA, middle cerebral artery; PRP, platelet-rich plasma; TTC, triphenyltetrazolium chloride. 
in vitro, does not appear to be effective in clinical trials of stroke (18). However, the inhibition of platelet aggregation provided by these agents is incomplete, so that these studies do not negate a potentially useful effect of more selective and more potent antiplatelet agents.

To test the hypotheses that in situ microvascular thrombosis occurs in stroke and contributes to ischemic neuronal injury, a murine model of focal cerebral ischemia and reperfusion was used to $(a)$ establish the presence and location of microvascular thrombus formation, and $(b)$ to demonstrate that inhibiting platelet aggregation can augment postischemic cerebral perfusion and thereby limit infarct size. To test the second part of this hypothesis, we tested the effectiveness of a potent agent (SDZ GPI 562), which blocks GP IIb/IIIa-mediated interplatelet/fibrinogen bridging and thereby limits platelet aggregation and thrombus accumulation.

\section{Methods}

\section{Murine stroke model}

Procedure. Mice (C57B1/6J, male) were subjected to stroke according to previously published procedures (4) and in accordance with guidelines of the American Academy of Accreditation of Laboratory Animal Care. After anesthesia with $0.3 \mathrm{ml}$ of ketamine $(10 \mathrm{mg} / \mathrm{ml}, \mathrm{IP})$ and xylazine $(0.5 \mathrm{mg} / \mathrm{ml}, \mathrm{IP})$, the carotid artery was accessed using the operative approach previously described in detail (4), including division/coagulation of the occipital and pterygopalatine arteries to obtain improved visualization and vascular access. A nylon suture was then introduced into the common carotid artery, and threaded up the internal carotid artery to occlude the origin of the right middle cerebral artery (MCA). Nylon (polyamide) suture material was obtained from United States Surgical Corporation (Norwalk, CT), and consisted of 5.0 nylon/13 mm length for 27-36 g mice, and 6.0 nylon/12 $\mathrm{mm}$ length for 22-26 g mice. After $45 \mathrm{~min}$ of occlusion, the suture was withdrawn to achieve a reperfused model of stroke. Although no vessels were tied off after the suture was removed, the external carotid arterial stump was cauterized to prevent frank hemorrhage. Measurements of relative cerebral blood flow were obtained as previously reported (4-6), using a straight laser doppler flow probe placed $2 \mathrm{~mm}$ posterior to the bregma, and $6 \mathrm{~mm}$ to each side of the midline using a stereotactic micromanipulator, keeping the angle of the probe perpendicular to the cortical surface. These cerebral blood flow measurements, expressed as the ratio of ipsilateral to contralateral blood flow, were obtained at baseline, immediately before MCA occlusion, 45 min after MCA occlusion, and at several time points after withdrawal of the occluding suture.

Measurement of cerebral infarction volumes. After $24 \mathrm{~h}$, animals were killed and their brains rapidly harvested. Infarct volumes were determined by staining serial cerebral sections with triphenyltetrazolium chloride (TTC) and performing computer-based planimetry of the negatively stained areas to calculate infarct volume (using National Institutes of Health image software).

Neurological exam. Before giving anesthesia, mice were examined for neurological deficit $23 \mathrm{~h}$ after reperfusion using a four-tiered grading system: a score of 1 was given if the animal demonstrated normal spontaneous movements; a score of 2 was given if the animal was noted to be turning towards the ipsilateral side; a score of 3 was given if the animal was observed to spin longitudinally (clockwise when viewed from the tail); and a score of 4 was given if the animal was unresponsive to noxious stimuli. This scoring system has been previously described in mice $(4,5)$, and is based upon similar scoring systems used in rats (19).

\section{Use of a GP IIb/IIIa antagonist}

Animals were treated with the indicated doses (and at the indicated times) with either an intravenous injection (given via the penile vein) of physiological saline or with physiological saline to which SDZ GPI 562 (GPI 562, Novartis Pharmaceuticals, Basel, Switzerland) had been added at the indicated concentrations. GPI 562 is a potent orally active and direct-acting nonpeptidic GP IIb/IIIa antagonist (20) of which the structure is shown in Fig. 1. In vitro, GPI 562 is a potent inhibitor of fibrinogen binding to purified GP IIb/IIIa (IC50 0.23 $\mathrm{nM}$ ), fibrinogen binding to platelets in whole blood (IC50 $1.7 \mathrm{nM}$ ), and platelet aggregation induced by various agonists (IC50 $\sim 11-55$ $\mathrm{nM})$. The antiplatelet effect of GPI 562 is species-specific, human and monkey platelets being inhibited most potently followed in decreasing order of potency by guinea pig, dog, minipig, hamster, and rabbit platelets. GPI 562 is specific for GP IIb/IIIa, showing negligible affinity for related integrins, such as the vitronectin receptor $\alpha_{V} \beta_{3}$, the fibronectin receptor $\alpha_{5} \beta_{1}$, or $\beta_{2}$ integrins, as determined by ligand binding to receptor or cell adhesion experiments. The binding of several ligands to GP IIb/IIIa (i.e., fibronectin, vitronectin, and vWf) is inhibited with the same potency. When administered to guinea pigs, dogs, or rhesus monkeys, GPI 562 dose dependently inhibits ex vivo platelet aggregation after intravenous, intraduodenal, or oral administration. Unlike most other orally active GP IIb/IIIa antagonists, SDZ GPI 562 is not a prodrug, thus obviating the need for metabolic conversion after oral administration. Because of poor bioavailability after oral administration in mice, the current experiments were performed using intravenous dosing.

\section{Measurement of bleeding time}

Bleeding times were measured in mice that were not subjected to experimental manipulation other than by receiving either vehicle (saline) or GPI 562 prepared in physiological saline and administered intravenously $5 \mathrm{~min}$ before the experiment. After anesthesia, a standardized incision was made on the central dorsal tail vein, and the tail was then immersed in physiological saline at $37.5^{\circ} \mathrm{C}$. Time was recorded from the moment blood was observed to emerge from the wound until cessation of blood flow (21).

\section{Measurements of cerebral thrombosis}

Separate cohorts of mice were used for each of the following experiments.

${ }^{111}$ In-platelet accumulation. Platelet accumulation was determined using ${ }^{111}$ In-labeled platelets, collected and prepared as previously described $(22,23)$. In brief, pooled blood was collected from control mice in $3.8 \%$ sodium citrate for anticoagulation (10 $\mathrm{ml}$ total). Platelets were isolated by differential centrifugation, first at $300 \mathrm{~g}$ for $5 \mathrm{~min}$ to obtain platelet-rich plasma, which was then washed three times at $2,000 \mathrm{~g}$ for $15 \mathrm{~min}$ in $10 \mathrm{ml}$ of acid/citrate/dextrose anticoagulant (ACD-A, containing $38 \mathrm{mmol} /$ liter citric acid, $75 \mathrm{mmol} /$ liter sodium citrate, and $135 \mathrm{mmol} /$ liter glucose). The pellet was suspended in $5 \mathrm{ml}$ of ACD-A and centrifuged at $100 \mathrm{~g}$ for $5 \mathrm{~min}$ to remove contaminating red blood cells, and the supernatant was collected. ${ }^{111}$ In-oxyquinoline $(70 \mu \mathrm{l}$ of $1 \mathrm{mCi} / \mathrm{ml}$; Amersham Mediphysics, Arlington Heights, IL) was added, and the suspension was shaken gently for $30 \mathrm{~min}$ at room temperature. The radiolabeled platelets were washed three

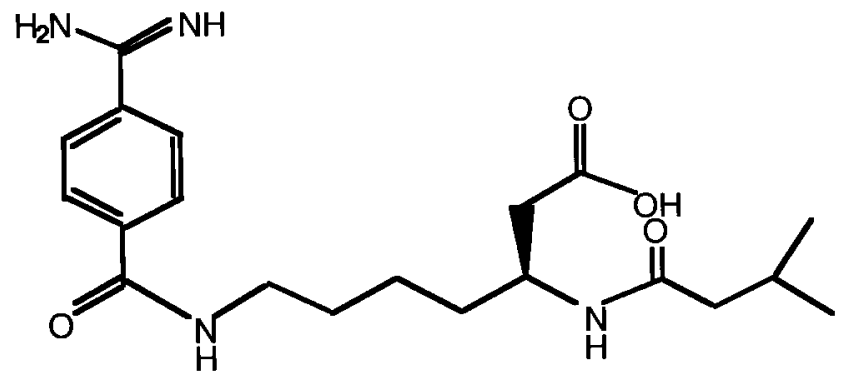

Figure 1. The chiral chemical structure of SDZ GPI 562, a GP IIb/ IIIa inhibitor, is shown. 
times in ACD-A and resuspended in PBS, and the platelet number was adjusted to $5 \times 10^{6} / \mathrm{ml}$. (10 counts were given to each animal). Immediately before insertion of the occluding suture, $0.2 \mathrm{ml}$ of ${ }^{111} \mathrm{In}$ labeled platelet suspension was injected intravenously into the penile vein; at $24 \mathrm{~h}$ of reperfusion, brain tissue was harvested and platelet accumulation was quantified as the ipsilateral/contralateral cpm ratio.

Detection of intracerebral fibrin. Mice were first subjected to focal cerebral ischemia and reperfusion as described above. To detect fibrin by either immunostaining or immunoblotting, mice were heparinized $(1,000 \mathrm{U} / \mathrm{ml}, 0.2 \mathrm{ml}$ given intravenously) $\sim 1 \mathrm{~min}$ before death) to minimize postmortem thrombosis. After separation into right and left hemispheres, immunoblotting for fibrin was performed as described previously (8). Because fibrin is extremely insoluble, brain tissue extracts were prepared by plasmin digestion, and plasmin-digested extracts were applied to a reduced 10\% SDS-polyacrylamide gel (10 mg protein/lane) for subsequent electrophoresis. After electrophoretic transfer to a nitrocellulose membrane, immunoblotting was performed using a primary polyclonal rabbit antihuman antibody prepared to $\gamma-\gamma$ chain dimers present in cross-linked fibrin (generously provided by J. Sobel; this antibody can detect murine fibrin [8], with relatively little cross-reactivity with fibrinogen [24]). After application of a secondary antibody (horseradish-peroxidase-conjugated goat anti-rabbit IgG), the enhanced chemiluminescent method (Amersham, Buckinghamshire, England) was used to detect the location of the fibrin bands. Using National Institutes of Health Image and Adobe Photoshop software, the relative intensity of the ipsilateral and contralateral bands were compared. In separate experiments, whole brains were recovered after stroke/heparinization, fixed in $10 \%$ formalin, embedded in paraffin, sectioned, and immunostained using a rabbit antibody raised to rat fibrin monomer (anti-rat fibrin IgG, $50 \mathrm{mg} / \mathrm{ml}$, provided by Jeff Emeis) (25), which is also cross-reactive with murine fibrin (8). Sites of primary antibody binding were visualized with a peroxidase-conjugated secondary antibody, goat-anti-rabbit IgG (Sigma Chemical Co., St. Louis, MO).

\section{Measurement of intracerebral hemorrhage (ICH)}

ICH was quantified using a spectrophotometric assay for hemoglobin, which has been recently developed and validated for use in a murine model of stroke (26). In brief, mouse brains were homogenized, sonicated, centrifuged, and hemoglobin in the supernatants was converted (with Drabkin's reagent) to cyanomethemoglobin, of which the concentration was assessed by measuring OD at $550 \mathrm{~nm}$ against a standard curve generated with known amounts of hemoglobin. Visual scoring of ICH was performed by a blinded observer on $1 \mathrm{~mm}$ serial coronal sections based on maximal hemorrhage diameter seen on any of the sections (ICH score 0 , no hemorrhage; $1,<1 \mathrm{~mm} ; 2,1-2 \mathrm{~mm}$; $3,2-3 \mathrm{~mm} ; 4,>3 \mathrm{~mm})$. This latter method has also been recently described (26).

\section{Measurement of platelet aggregation}

Platelet aggregation (and its inhibition by GPI 562) was measured in both an in vitro assay as well as in mice ex vivo. Platelet-rich plasma (PRP) was obtained from pooled blood from 10 control mice $(7.5 \mathrm{ml}$ total), anticoagulated with $750 \mu \mathrm{l}$ heparin $(1,000 \mathrm{U} / \mathrm{ml})$, divided in two $10 \mathrm{ml}$ polystyrene tubes $(\sim 4 \mathrm{ml} /$ tube $)$ and centrifuged for $10 \mathrm{~min}$ at 1,000 rpm. The supernatant and interphase PRP were transferred to fresh tubes, mixed, and the number of platelets was counted on a Symex PL-100 counter. Platelets were diluted to reach the final concentration of $10^{9}-1.5 \times 10^{9}$ platelets $/ \mathrm{ml}$. Aggregation experiments were performed in a dual channel Payton aggregometer with a Rikadenki recorder connected. PRP $(150 \mathrm{ml})$ was placed in a cuvette containing a steel stirring bar, and plasma from GPI 562-treated mice $(170 \mathrm{ml})$ was added and stirred $(1,100 \mathrm{rpm})$ at $37^{\circ} \mathrm{C}$ for $1 \mathrm{~min}$ before the addition of $20 \mathrm{ml}$ of agonist solution (ADP $17 \mathrm{mmol} /$ liter, to reach a final concentration of $1 \mathrm{mmol} /$ liter). After $2 \mathrm{~min}$, platelet aggregation was quantified as a function of the change in light transmission through the aggregometry cuvette. Whole blood platelet aggregation was determined as described (27). Briefly, platelet counts were deter- mined in whole blood on a cell counter (Medonic-Servotec CA-600) before, and 1 and 2 min after addition of the platelet agonist (ADP) to the blood to which GPI 562 had been added. For these experiments, GPI 562 was administered intravenously at the indicated doses, and blood withdrawn for subsequent analysis.

\section{Data analysis}

Values are expressed as means \pm SEM, with the numbers of experiments performed provided in the figure legends. For experiments in which two variables were compared, unpaired Student's $t$ test was used. For experiments in which more than two variables were compared, one way ANOVA was used, with Tukey's procedure used to test for significant differences. Contingency analysis using Fisher's exact test was performed to test for differences in mortality between various treatments. Data were considered significantly different when $P<0.05$.

\section{Results}

To test the hypothesis that thrombosis occurs in the region of the brain subjected to ischemia, the accumulation of both platelets and fibrin was examined, because these are integral components of the developing thrombus. To quantify platelet accumulation, a strategy was used wherein mice subjected to cerebral ischemia and reperfusion by withdrawal of the MCAoccluding suture were injected with radiolabeled platelets immediately before the MCA occlusion. The accumulation of ${ }^{111}$ In-labeled platelets at $24 \mathrm{~h}$ of reperfusion was quantified as the ipsilateral (right) to contralateral (left) hemispheric cpm. In animals which received the radiolabeled platelets but no surgical procedure (Fig. 2 A, left bar), as expected, the ipsilateral to contralateral accumulation was nearly equal (ratio $\sim 1.0$ ). In contrast, when mice were subjected to focal cerebral ischemia and reperfusion, there was nearly a threefold increase in platelet accumulation in the ipsilateral (postischemic) hemisphere compared with the contralateral nonischemic hemisphere (Fig. 2 A, middle bar).

Using an antibody prepared against rat fibrin monomer (25) in an immunoblotting technique to detect fibrin, which has been previously published (8), the accumulation of fibrin was examined in plasmin-digested extracts of postischemic brain tissue. These experiments (Fig. $2 \mathrm{~B}$, right two lanes) demonstrated a distinct ( $\sim$ threefold by densitometric analysis) increase in the accumulation of fibrin in the ipsilateral hemisphere. Immunohistochemistry demonstrated that the predominant site of fibrin accumulation was within the ipsilateral cerebral microvasculature (Fig. $2 C$, top).

These data, as well as previously published studies, suggested the possibility that the accumulation of thrombus in the ipsilateral microvasculature may have a pathogenic role in cerebral ischemia/reperfusion injury. To test this hypothesis, a novel nonpeptidic GP IIb/IIIa inhibitor (GPI 562) was tested with respect to its ability to inhibit the accumulation of thrombus in the postischemic cerebral territory. To first characterize the efficacy of this agent to inhibit in vitro platelet aggregation, the ability of GPI 562 to inhibit murine platelet aggregation was first tested with known agonists of platelet aggregation. Of all of the agonists tested (ADP, epinephrine, norepinephrine, serotonin, collagen, and arachidonic acid), ADP showed the strongest platelet aggregation activity, reaching its maximal effect at $1-3 \mu \mathrm{mol} /$ liter (H.G. Zerwes, unpublished data). Experiments were then performed with ADP to determine the efficacy of GPI 562 to inhibit murine platelet aggregation. ADP (1 


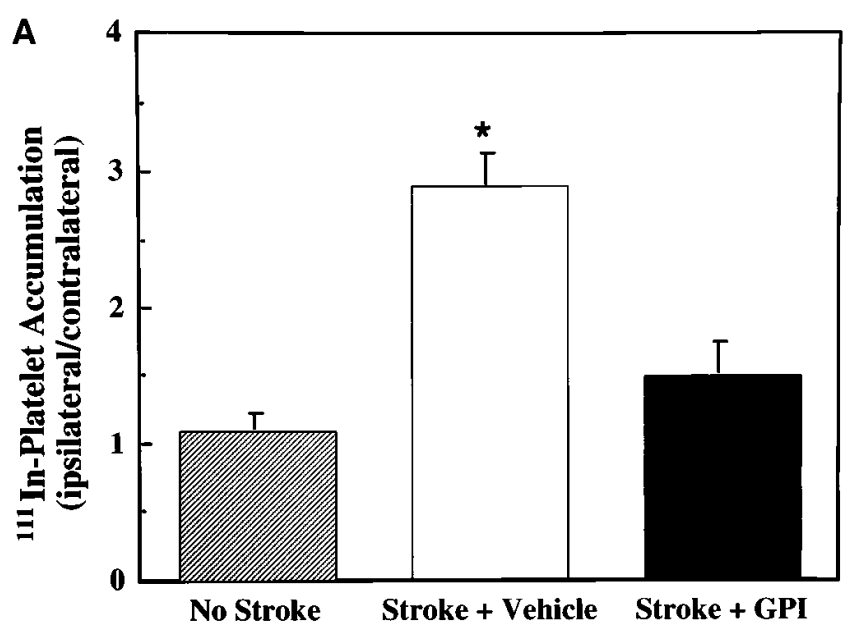

C

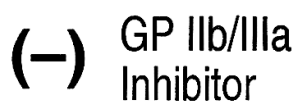

$(+)$
$\mathrm{GP} \| \mathrm{lb} / \mathrm{lla}$ Inhibitor
Ipsilateral
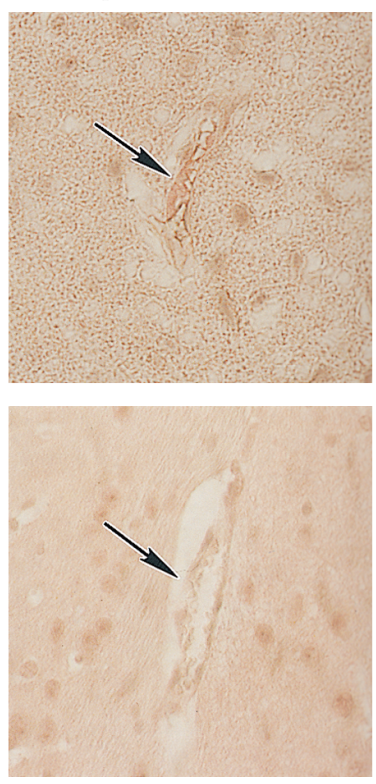

\section{Contralateral}
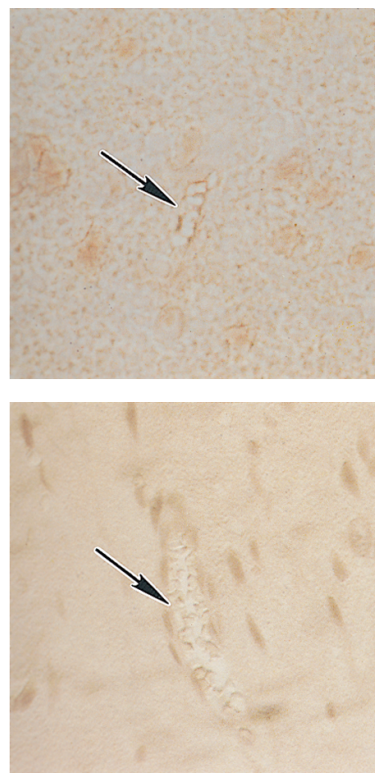

$(+)$ GPIIb/IIla Inhibitor

\section{Fibrin $\rightarrow$}

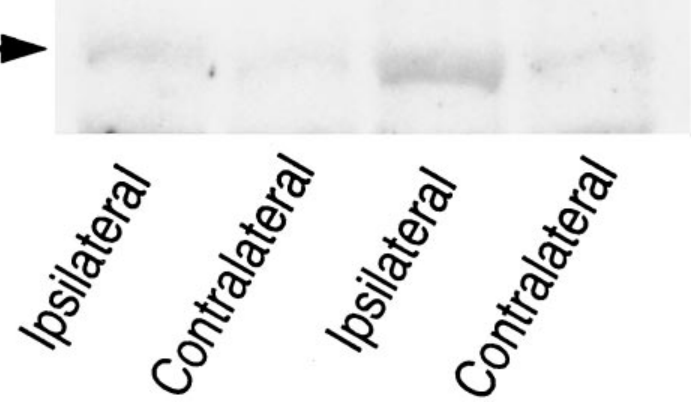

$(-)$ GPIIb/Illa Inhibitor

Figure 2. Effect of stroke with or without GP IIb/ IIIa inhibition on platelet and fibrin accumulation in the brain. $(A)^{111}$ In-labeled platelets were administered to control mice ("No Stroke," $n=11$ ), and $24 \mathrm{~h}$ later, brains were harvested, divided into right and left hemispheres, and counted. Relative platelet accumulation is expressed as the ratio of right/left hemispheric cpm. Using similar techniques with ${ }^{111}$ In-platelet administration immediately before the 45-min period of MCA occlusion, relative platelet accumulation was measured at $24 \mathrm{~h}$ after reperfusion (Stroke + vehicle [saline], $n=10$, expressed as the ipsilateral [right]/contralateral[left] cpm). In mice subjected to identical MCA occlusion/reperfusion procedures, the effect of a GP IIb/IIIa inhibitor (GPI 562, $5 \mathrm{mg} / \mathrm{kg}$ given before MCA occlusion, $n=10$ ) on platelet accumulation was recorded. Data are shown as means \pm SEM, with * $=P<0.005$ versus No Stroke and versus Stroke + GPI. $P=$ NS between No Stroke and Stroke + GPI. $(B)$ The accumulation of fibrin at $24 \mathrm{~h}$ was measured in mice pretreated with vehicle ([-] GP IIb/IIIa inhibitor) or GPI $562(5 \mathrm{mg} / \mathrm{kg},[+]$ GP IIb/IIIa inhibitor $)$ and subjected to MCA occlusion/reperfusion. At $24 \mathrm{~h}$, brain tissue was divided into ipsilateral and contralateral hemispheres, and subjected to immunoblotting using a primary antibody which recognizes a neoepitope on the $\gamma-\gamma$ chain dimer of cross-linked fibrin. $(C)$ Immunostaining for fibrin in the contralateral (top right) and ipsilateral (top left) cerebral hemispheres $24 \mathrm{~h}$ after stroke. Microvessels are indicated with arrows. Intravascular fibrin formation can be seen as red staining in the postischemic microvasculature. When GPI $562(5 \mathrm{mg} / \mathrm{kg})$ was administered before stroke, there was an apparent reduction in microvascular fibrin accumulation at $24 \mathrm{~h}($ bottom).

$\mu \mathrm{mol} / \mathrm{liter}$ ) was added to murine whole blood or to plateletrich plasma (PRP). When administered ex vivo, GPI 562 inhibited ADP-induced platelet aggregation in a dose-dependent fashion, with $\mathrm{IC}_{50} \mathrm{~s}$ of $1.1 \times 10^{-5} \mathrm{~mol} / \mathrm{liter}$ and $3.6 \times 10^{-6} \mathrm{~mol} / \mathrm{li}-$ ter, for whole blood and PRP, respectively. (Fig. $3 A$; the GPI 562 dose-dependent inhibition on ex vivo ADP-induced platelet aggregation in PRP is shown). Because of the lower variability between measurements when performed on PRP rather than whole blood, the effects of intravenous doses of GPI 562 were examined with respect to their ability to inhibit platelet aggregation in PRP. In these experiments (in vivo administration of GPI 562 followed by ex vivo platelet studies), intravenous GPI 562 was also shown to inhibit platelet aggregation in a dose-dependent fashion (Fig. 3 B). As can be seen, GPI 562 injected at the dose of $0.5,1.0,2.0$, and $5.0 \mathrm{mg} / \mathrm{kg}$ inhibited dose related to the ADP-induced platelet aggregation 5 min after intravenous injection, with an $\mathrm{IC}_{50}$ of $1.4 \mathrm{mg} / \mathrm{kg}$. This effect of GPI 562 to inhibit murine platelet aggregation ex vivo was no longer present $1 \mathrm{~h}$ after administration, suggesting that the inhibitory effects of intravenously administered GPI 562 on platelet-aggregation are short-lasting in the mouse. As a final experiment to test the in vivo effects of GPI 562 on tail vein bleeding time, a standardized model was performed in which the tail was immersed in constant temperature physiological saline after a standardized incision, and time to cessation of bleeding recorded (21). These data showed a GPI 562 dosedependent increase in bleeding time in mice after intravenous administration (Fig. $3 C$ ). 


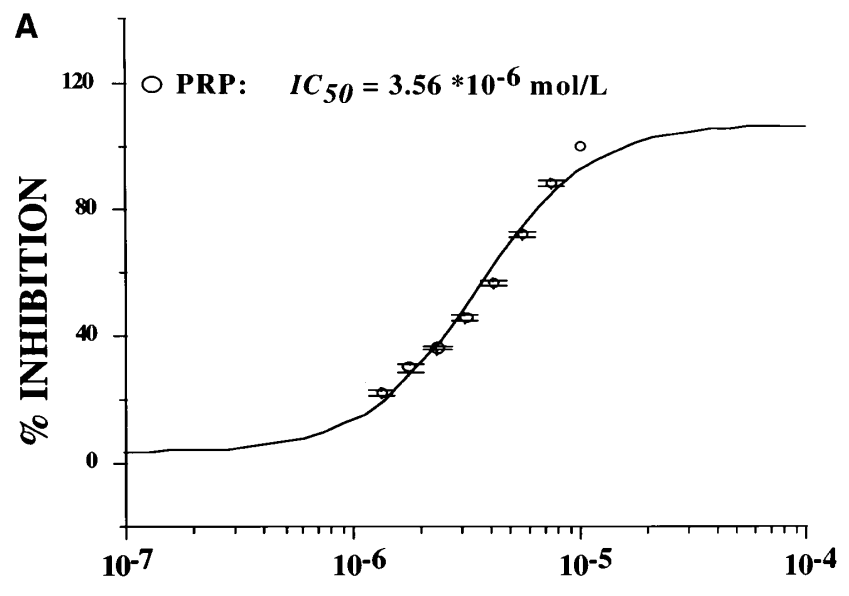

GPI $562(\mathrm{~mol} / \mathrm{L})$

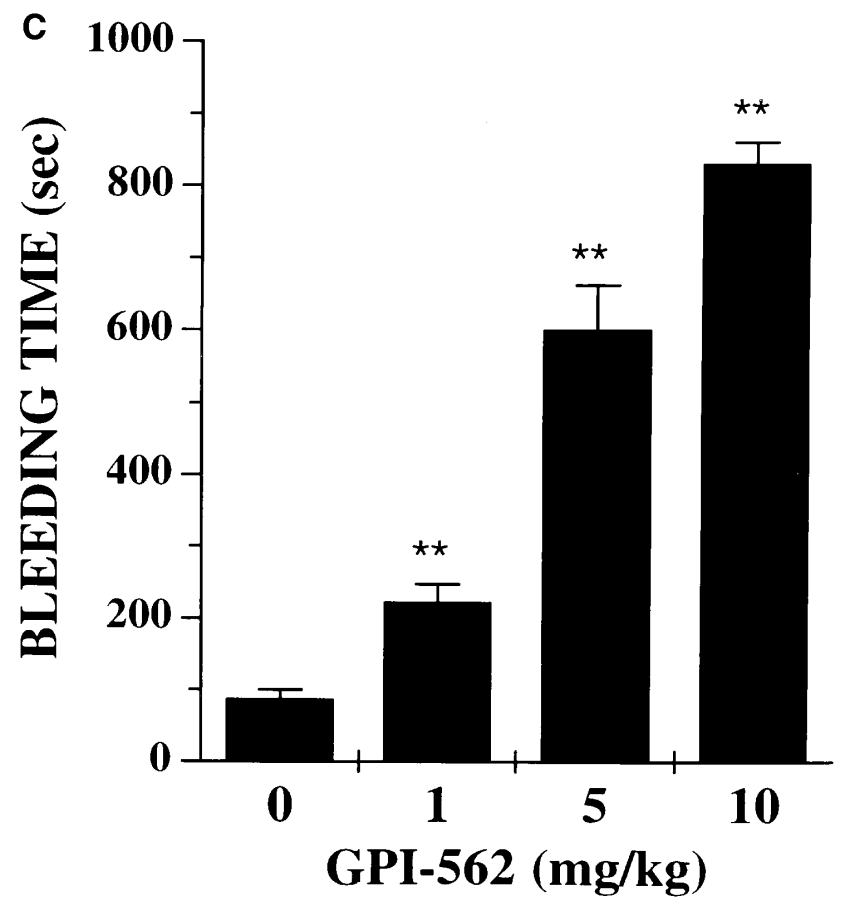

To test whether GPI 562 could effectively reduce cerebral thrombosis in stroke, an intravenous dose $(5 \mathrm{mg} / \mathrm{kg})$ was given to mice before middle cerebral artery occlusion. This treatment caused a significant reduction in the relative accumulation of platelets in the ipsilateral hemisphere (Fig. $1 \mathrm{~A}$, right bar). This relative accumulation of platelets in the ipsilateral hemisphere of GPI 562-treated animals did not differ significantly from animals that had not been subjected to stroke. In concordance with the data demonstrating the ability of GPI 562 to reduce ipsilateral platelet accumulation, the relative accumulation of fibrin in the ipsilateral hemisphere was also reduced, by $\sim 50 \%$, by pretreatment of animals with GPI 562 (Fig. $1 \mathrm{~B}$, left two lanes). This immunoblot data was confirmed by immunohistochemical data showing a relative paucity of intravascular fibrin in the GPI 562-treated animals (Fig. 2 C, bottom two panels).

The next set of experiments was performed to demonstrate

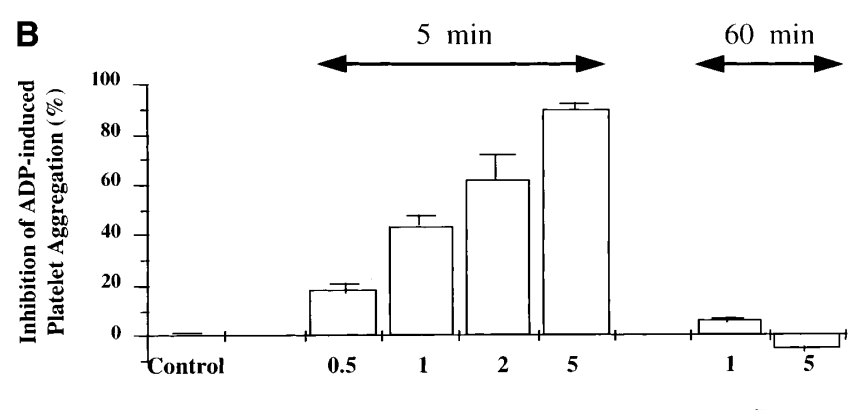

Intravenous GPI 562 Dose (mg/kg)

Figure 3. Effect of GPI 562 on platelet aggregation and bleeding time. $(A)$ Effect of ex vivo administration of GPI 562 on platelet aggregation. Using platelet-rich plasma $(P R P)$ and the indicated concentrations of GPI 562, platelet aggregation was measured in a magnetically stirred aggregometry cuvette, after challenge with ADP ( $1 \mathrm{mmol} /$ liter $)$. The $\mathrm{IC}_{50}$ is shown. (B) Effect of intravenous administration of GPI 562 (doses indicated) on inhibition of platelet aggregation in PRP prepared 5 or 60 min after GPI 562 administration. $N=5$ mice per dose, means \pm SEM are shown. (C) Effect of GPI 562 administration on tail vein bleeding time. GPI 562 was administered intravenously at the indicated doses, and the tail vein bleeding time was determined as described in the Methods section. $N=5$ animals per time point, $* *=P<$ 0.01 versus the control (vehicle only, $0 \mathrm{mg} / \mathrm{kg}$ ).

whether these observations regarding the occurrence of cerebral microvascular thrombosis and its inhibition with GPI 562 could be pathophysiologically relevant in the setting of murine stroke. To test this, a range of doses of GPI 562 was administered to different cohorts of animals before MCA occlusion, and cerebral infarct volumes were measured by TTC staining of serial cerebral sections. These experiments indicate that both the 1 and $5 \mathrm{mg} / \mathrm{kg}$ doses were highly effective at reducing cerebral infarct volumes (Fig. 4). Because microvascular thrombosis is likely to reduce postischemic cerebral blood flows, the effects of graded doses of GPI 562 on postischemic cerebral blood flow were examined. Cerebral blood flow, measured by laser doppler and expressed as the ipsilateral/contralateral flow ratio, was significantly improved in GPI 562treated animals (Fig. 5). In addition to examining the effects of GP IIb/IIIa inhibition on cerebral thrombosis, blood flow, and infarction volume, $24 \mathrm{~h}$ after stroke (before anesthesia), the 


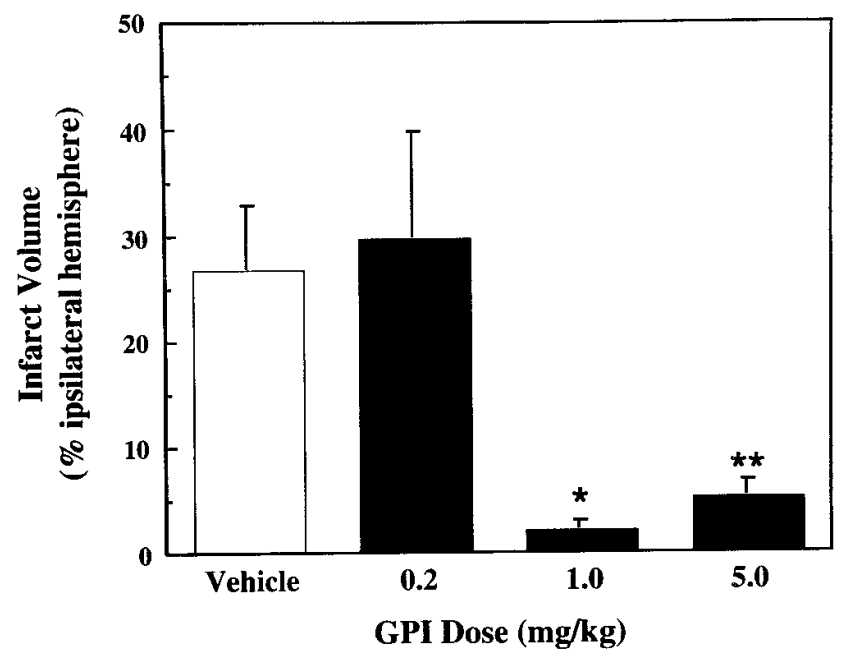

Figure 4. Effect of an inhibitor of GP IIb/IIIa on cerebral infarct volumes after MCA occlusion and reperfusion. GPI 562 was administered intravenously before MCA occlusion at the indicated doses, and infarct volumes were calculated from TTC-stained serial cerebral sections. $N=31,5,7$, and 11 for the vehicle, $0.2,1.0$, and $5.0 \mathrm{mg} / \mathrm{kg}$ doses, respectively. Data are shown as means \pm SEM, $*=P<0.05$ versus vehicle, and $* *=P<0.01$ versus vehicle.

mice were scored for neurological deficit as described in Methods (a score of 4 representing the greatest neurological impairment); although differences were not statistically different between groups, a slight tendency for improvement seen at the 1 and $5 \mathrm{mg} / \mathrm{kg}$ dose disappeared with the $10 \mathrm{mg} / \mathrm{kg}$ dose (Fig. 6).

Although a $10 \mathrm{mg} / \mathrm{kg}$ dose was also tried, there was substantial $(75 \%)$ perioperative mortality, with the subjective observation of increased bleeding during surgery. Although there was a dose-dependent increase in perioperative mortality when GPI 562 was given before surgery (Fig. $7 A$ ), overall mortality did not differ significantly between any of the doses tested when GPI 562 was administered preoperatively (Fig. 7 $C$ ), perhaps because the postoperative mortality tended to be lower in these animals (Fig. 7 B). When GPI 562 was given after reperfusion, there was a $45 \%$ reduction in overall mortality, compared with corresponding vehicle-treated controls $(P<0.05)$. Taken together, these mortality data suggest that GPI 562 may induce procedure-related complications which may not be relevant to clinical stroke.

Because these data suggested that the use of a GP IIb/IIIa inhibitor, such as GPI 562, might exacerbate cerebral hemorrhage in stroke, we used two complementary methods for assessing intracerebral hemorrhage in stroke (26). In the first, photographic images were obtained of serial coronal sections at $24 \mathrm{~h}$ after stroke. Intracerebral hemorrhage was graded by an observer blinded to the treatment regimen using a scoring system based upon maximal hemorrhagic diameter in serial cerebral sections, with 4 representing maximal hemorrhagic area (Fig. $8 A$ ). In a second complementary method to detect $\mathrm{ICH}$, a recently validated (26) spectrophotometric assay was used to quantify the amount of ICH after stroke (Fig. $8 \mathrm{~B}$ ). Both methods revealed a significant increase in ICH only at the $10 \mathrm{mg} / \mathrm{kg}$ dose.

Taken together, these data indicate that, when given before MCA occlusion, inhibition of cerebral microvascular thrombosis by a strategy of GP IIb/IIIa blockade reduces tissue injury

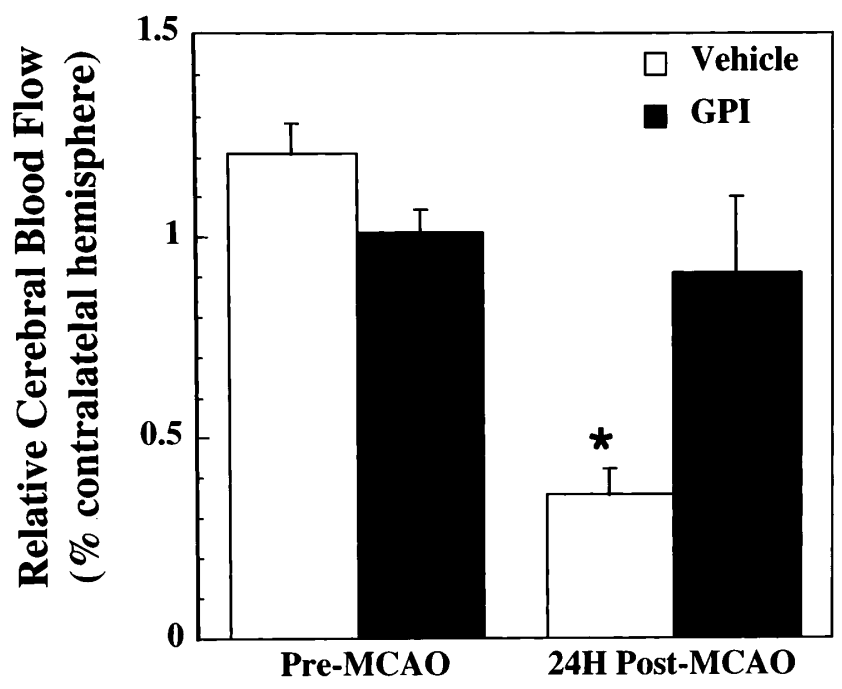

Figure 5. Relative cerebral blood flow after stroke. 15 min before middle cerebral artery occlusion (time 0 ), relative cerebral blood flow to the ipsilateral hemisphere was recorded from precisely defined neuroanatomical landmarks (see Methods) (4), and expressed as the ratio of doppler flow between ipsilateral and contralateral hemispheres. Recordings were also taken at $24 \mathrm{~h}$. GPI $562(5.0 \mathrm{mg} / \mathrm{kg})$ was administered intravenously before MCA occlusion in 11 animals; 31 animals were given vehicle as a control. Data are shown as means \pm SEM, $*=P<0.05$

in stroke. However, as patients who present with acute stroke do so after the onset of cerebral ischemia, experiments were performed to test whether GPI 562 would be effective if given after the onset of cerebral ischemia. Using the $5 \mathrm{mg} / \mathrm{kg}$ dose, infarct volume analysis reveals that GPI 562 reduces infarct volumes even when given after middle cerebral artery occlusion (Fig. 9), without increasing intracerebral hemorrhage (Fig. 10).

\section{Discussion}

The data presented here indicate that microvascular thrombi (both platelets and fibrin) accumulate in the ipsilateral microvasculature in the setting of stroke. Not only is ipsilateral thrombosis reduced when platelet integrin GP IIb/IIIa-mediated platelet aggregation is inhibited using GPI 562, but there is a concomittant reduction in cerebral infarct volumes and an improvement in postischemic cerebral blood flow. Although platelet inhibition has shown some efficacy in the primary prevention of stroke (28), the effectiveness of GP IIb/IIIa antagonism has not been reported in either primary prevention of stroke or in the treatment of acute stroke in progress. Because GP IIb/IIIa antagonism is effective to prevent local thrombosis in a canine carotid injury model (29) and to reduce plateletmediated cyclical blood flow reductions in a primate carotid thrombosis/endothelial injury model (30), we hypothesized that it may also be effective to inhibit microvascular thrombosis, which occurs distal to the obstruction site of a major cerebrovascular arterial tributary. The data in this paper demonstrate that GPI 562 is effective at reducing thrombosis in this distal vascular territory. Moreover, the ability of GP IIb/IIIa blockade to improve outcome when given after the onset of 


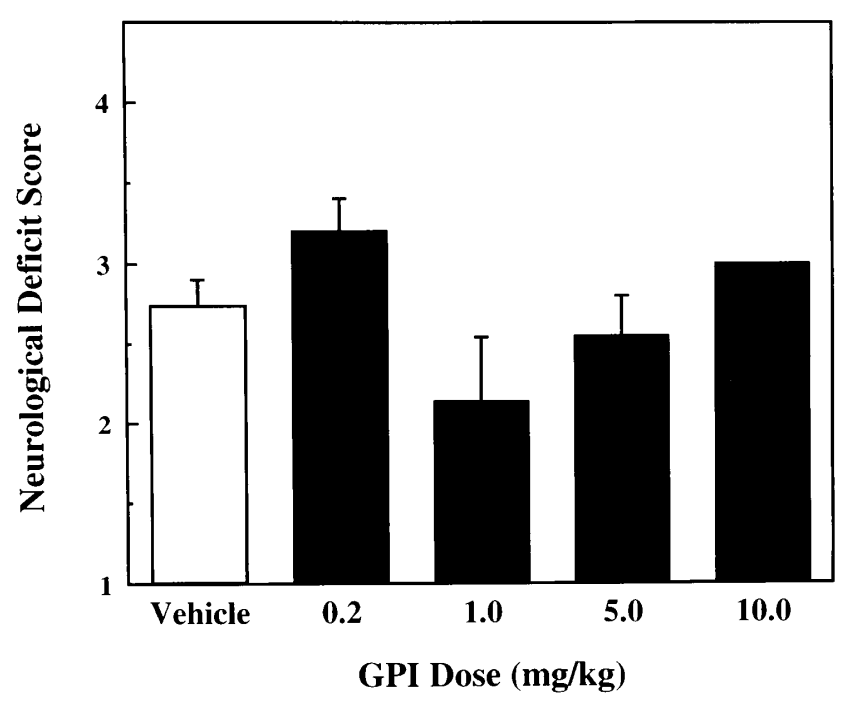

Figure 6. Neurological deficit scores of animals after stroke. The indicated doses of GP 562 were administered intravenously preoperatively, followed by $45 \mathrm{~min}$ of ischemia and $23 \mathrm{~h}$ of reperfusion. Neurological deficit was scored in surviving animals from 1-4 as described in Methods ( $4=$ most severe deficit). $N=31,5,7,11$, and 8 for the vehicle, $0.2,1.0,5.0$ and $10 \mathrm{mg} / \mathrm{kg}$ doses, respectively. Means \pm SEM are shown.

ischemia suggests the occurrence of an ongoing, dynamic process of microvascular thrombosis after stroke onset.

In the model used to create stroke for the experiments presented here, there is a potential for the use of the intravascular suture to cause endothelial injury and platelet activation, which may lead to some degree of artifactual elevation in intracerebral thrombosis and, therefore, an exaggerated responsiveness to antiplatelet therapy. However, this is an inherent technical limitation that is also likely to apply to other models of stroke in rodents. These other models, including embolization of thrombus to the MCA (31) or extraluminal occlusion of the MCA (32), require either ipsilateral intravascular instrumentation (31) or vascular compression (32), which could also lead to vascular damage and enhanced platelet reactivity. These manipulations could also potentially cause endothelial damage leading to more pronounced platelet reactivity. However, procedure-related intravascular damage is not likely to be a significant contributing factor to the pathophysiology of the mice in these experiments for the following reason. Experiments in which the suture was advanced to the point at which it occluded the origin of the middle cerebral artery (verified by laser doppler) and then immediately withdrawn did not result in cerebral infarction (D.J. Pinsky, personal observation). It is, therefore, apparent that the ischemic period (and not just the threading procedure) is required to create cerebral infarction. There is also clinical data indicating a role for platelet accumulation in human stroke, as $\beta$-thromboglobulin, platelet factor- 4 , and thromboxane $B_{2}$ levels are elevated in acute stroke in humans (33-35).

Platelets form an integral part of a thrombus in vivo, aggregating in response to a variety of prothrombotic agents as they become entrapped in the developing fibrin mesh. As an important effector arm to amplify the coagulation mechanism, platelets not only adhere to the injured vascular wall, but aggregate predominantly through interactions with vWf; (especially un-
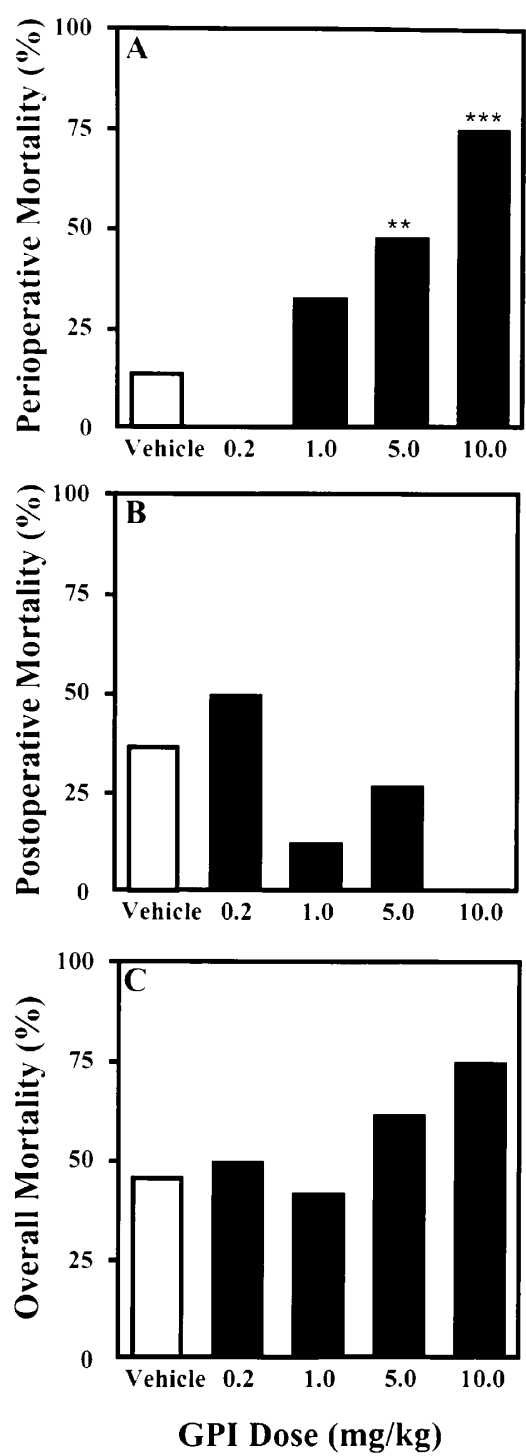

Figure 7. Dosedependent effects of a GP IIb/IIIa inhibitor on mortality. The indicated doses of vehicle or intravenous GPI 562 given preoperatively are shown. From left to right, the starting $n$ was $57,10,12,29$, and 8 , respectively. (A) Perioperative mortality (\%) is shown, and was defined as any death occurring within $90 \mathrm{~min}$ of surgery. (B) Postoperative mortality is shown, and was defined as any death occurring between the 90-min time point and the 24-h time point. (C) Overall mortality is shown. $* *=P<$ 0.01 versus vehicle.

der conditions of high shear) and fibrinogen (especially under lower shear conditions) (36). The initial adherence of platelets to the injured vascular wall is mediated by GP Ib and GP Ia/ IIa, which activates the platelets to induce a conformational change in GP IIb/IIIa receptors located in great abundance at the platelet surface $(37,38)$. GP IIb/IIIa, a member of the integrin family of heterodimeric glycoproteins, then mediates the calcium-dependent binding of fibrinogen (and vWf (39)) to platelets, which thereby aggregate in the process of thrombus formation. GP IIb/IIIa therefore serves as the final common pathway for platelet aggregation by mediating platelet/fibrinogen/platelet bridging. To examine the role of GP IIb/IIIa blockade in the treatment of stroke, we have used a highly selective GP IIb/IIIa antagonist, GPI 562, which is a peptidomimetic agent that acts upon the integrin RGD-binding site of platelets and exhibits 15,000-fold greater selectivity for GP IIb/ IIIa receptors than for fibronectin or vitronectin (20). In agreement with the studies shown here, this agent was shown to be a highly potent inhibitor of platelet aggregation in vitro, which diminished microthrombus formation in a model of discordant xenograft transplantation (40).

Because GP IIb/IIIa serves as the final common pathway 


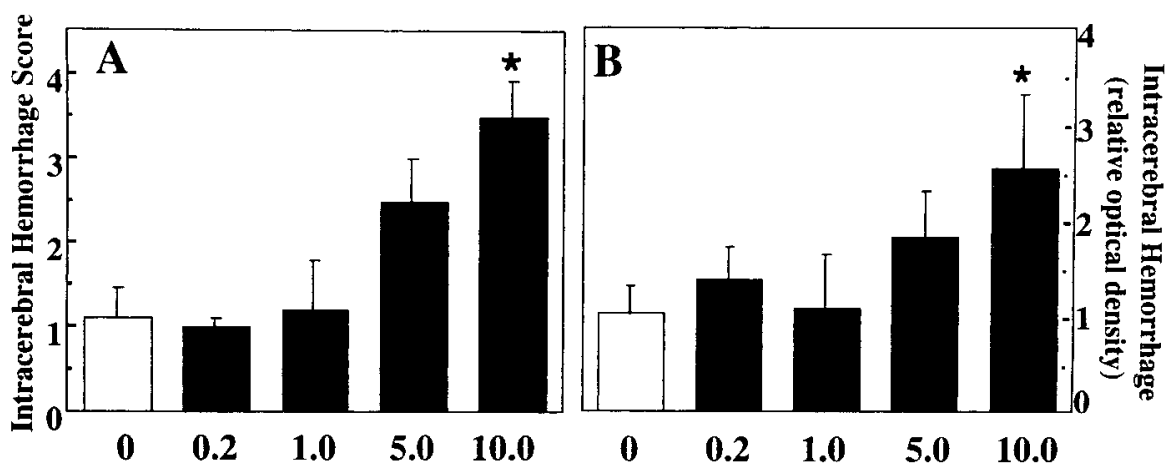

GPI Dose (mg/kg)
Figure 8. Effect of GP IIb/IIIa inhibition on intracerebral hemorrhage. $(A)$ Using a previously described scoring system (26), scored by an observer blinded to the treatment regimen, $\mathrm{ICH}$ was graded based upon maximal diameter of hemorrhage observed in serial cerebral sections. Scale $=1-4$, with 4 representing maximal hemorrhage. Means \pm SEM are shown. $*=P<0.05$ versus vehicle-treated controls. $(B)$ Using a recently validated spectrophotometric hemoglobin assay to quantify intracerebral hemorrhage (26), ICH was quantified at $24 \mathrm{~h}$ after MCA occlusion and reperfusion in animals pretreated intravenously with the indicated doses of GPI $562(n=11,3$, 5,6 , and 3 for bars from left to right). Data are expressed as the mean relative optical density (compared with vehicle-treated controls) \pm SEM, recorded at $550 \mathrm{~nm} ; *=P<$ 0.05 versus vehicle-treated controls. for platelet aggregation, blockade of these receptors provides a powerful target for antithrombotic therapy. In addition, there is the theoretical advantage that selective inhibition of GP IIb/IIIa, which does not interfere with the initial platelet adhesive event, may inhibit intravascular thrombosis, yet preserve extravascular hemostasis. This may be important in the setting of stroke, to reduce ICH even in the newly infarcted territory that is characterized by injured and friable vessels. When given in vitro, GP IIb/IIIa antagonists potently inhibit platelet aggregation after challenge with ADP, collagen, or thrombin $(41,42)$. In an in vivo baboon arteriovenous shunt

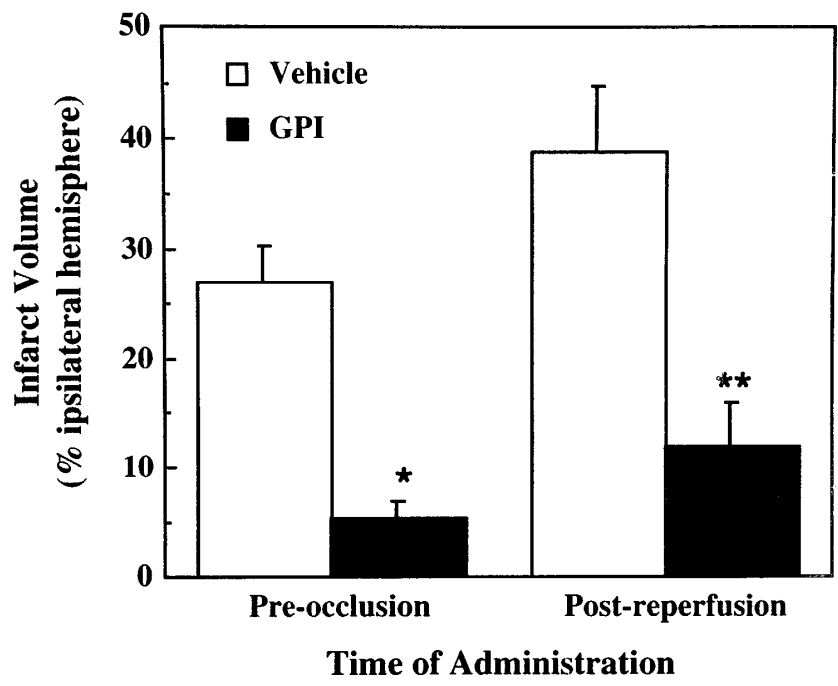

Figure 9. Effect of timing of administration of a GP IIb/IIIa inhibitor on cerebral infarct volumes in stroke. GPI $562(5 \mathrm{mg} / \mathrm{kg})$ was administered either immediately before MCA occlusion (pre-occlusion) or 45 min after MCA occlusion (post-reperfusion). The pre-occlusion data is identical to that shown in Fig. 3, displayed here for comparison with the same dose of GPI 562 given after reperfusion. Pre-occlusion: $n=$ 31 for vehicle, $n=11$ for GPI 562; Post-reperfusion: $n=7$ for control, and $n=15$ for GPI 562. Means \pm SEM are shown. $*=P<0.05$ versus pre-occlusion vehicle, and $* *=P<0.05$ versus post-reperfusion vehicle. model, a GP IIb/IIIa antagonist caused a potent and long-lasting effect to prevent arterial thrombosis (43). In a primate model of carotid artery thrombosis, anti-GP IIb/IIIa therapy decreased platelet-mediated cyclical flow reductions (30). In humans, anti-GP IIb/IIIa therapy with 7E3 (abciximab, a chimeric monoclonal $\mathrm{IgG} \mathrm{F}_{\mathrm{ab}}$ antibody fragment with high affinity to the GP IIb/IIIa receptor) has proven especially useful to reduce acute ischemic complications from percutaneous coronary intervention without increasing the rate of intracerebral hemorrhage (44). Several other clinical trials (45-48) have also confirmed the safety of GP IIb/IIIa antagonists and their efficacy to reduce ischemic complications in patients undergoing percutaneous coronary revascularization.

These findings suggest that GP IIb/IIIa blockade may prove useful in thrombotic disorders, such as ischemic stroke. However, to date, their usefulness in this setting has been suggested only by indirect experiments. In dogs, GP IIb/IIIa blockade prevents local thrombosis in a carotid injury model and improves carotid blood flow, cortical perfusion, and EEG tracings (29). An important ancillary observation in this study was that the use of a GP IIb/IIIa antagonist did not result in a significant increase in intracerebral hemorrhage at doses that exhibited clinical efficacy ( 1 and $5 \mathrm{mg} / \mathrm{kg}$ ), which is an important characteristic to be sought in any agent designed to treat stroke. However, there was an increase in ICH at the $10 \mathrm{mg} / \mathrm{kg}$ dose. Even though bleeding times do not necessarily accurately predict surgical bleeding (49), the possibility that higher doses of GPI 562 may increase procedure-related intracerebral hemorrhage is also supported by the tail vein bleeding time data, which demonstrate a GPI 562 dose-dependent increase in bleeding time. These observations, along with the increased perioperative (though lower postoperative) mortality and the subjective impression of increased bleeding in the surgical field at the $10 \mathrm{mg} / \mathrm{kg}$ dose, may help to explain the failure to detect overall mortality differences with preoperative GPI 562 treatment. However, when given immediately after reperfusion, there is a significant reduction in mortality and still no increase in intracerebral hemorrhage. Therefore, although the degree of intracerebral hemorrage may have been underestimated in GPI-treated animals due to dropouts from perioperative complications, this may not be relevant to clinical stroke, because 


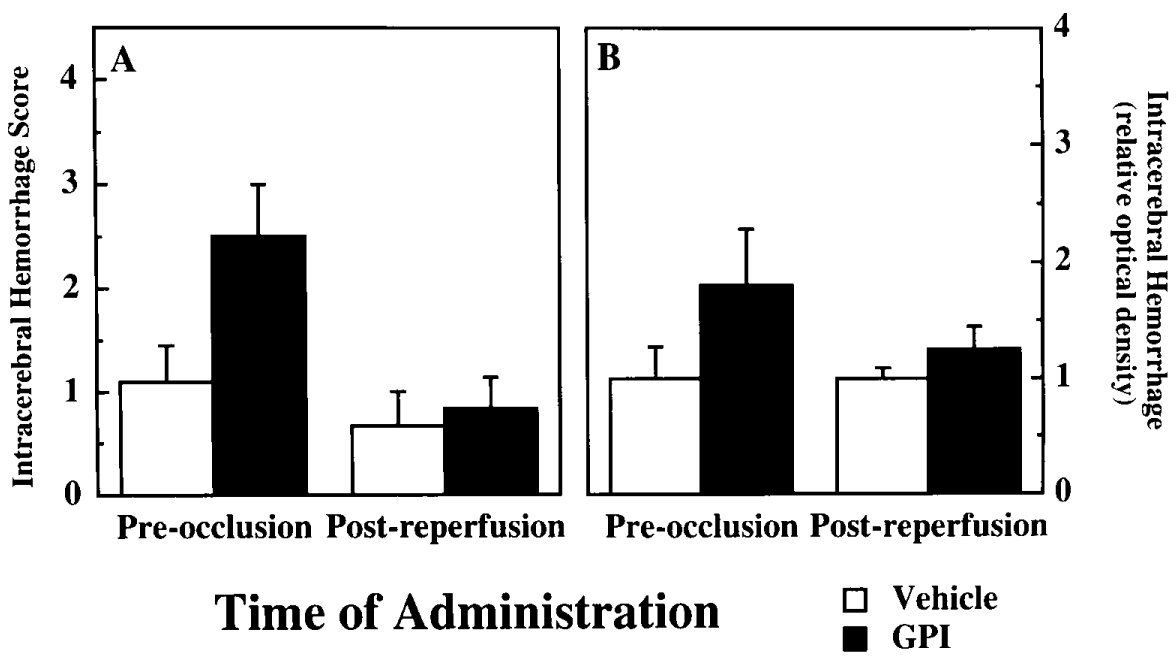

Figure 10. Effect of timing of GPI 562 administration on intracerebral hemorrhage. Either vehicle or GPI $562(5 \mathrm{mg} / \mathrm{kg})$ was given intravenously immediately before MCA occlusion (pre-occlusion) or immediately after withdrawal of the MCA-occluding suture (post-reperfusion), and the effects on intracerebral hemorrhage recorded as described in the legend to Fig. 8. The pre-occlusion data are the same as shown in the $5 \mathrm{mg} / \mathrm{kg}$ bar in Fig. 8 , but are repeated here to facilitate comparison with the same dose administered after reperfusion. $n=11$ and 6 for pre-occlusion vehicle and GPI 562, respectively, and $n=3$ and 6 for postischemic vehicle and GPI 562 administration, respectively. the method of stroke induction in these experiments is a model, which diverges from the clinical situation in stroke due to the de novo creation of a surgical wound.

Because most patients present with stroke during (rather than before) the ischemic phase, it was also necessary to demonstrate that the strategy of GP IIb/IIIa blockade might be effective when given the following onset of ischemia. When GPI 562 was given intravenously $(5 \mathrm{mg} / \mathrm{kg})$ after the onset of cerebral ischemia, the infarct volumes at $24 \mathrm{~h}$ are reduced $\sim 70 \%$ $(P=0.02)$ versus vehicle-treated controls. These data suggest that microvascular thrombosis does not occur as an instantaneous event at the time of occlusion of a major cerebrovascular tributary. Rather, microvascular thrombosis appears to be a dynamic, ongoing process after ischemia, because the antiplatelet agent tested was efficacious even when given after the ischemic phase of stroke in this murine model. In summary, these data indicate that platelets are key mediators in the pathogenesis of microvascular thrombosis and cerebral injury in stroke. The current studies suggest that inhibiting continuing thrombosis even after stroke onset, using a strategy of GP IIb/IIIa antagonism, may improve outcome when applied to patients as they typically present with stroke.

\section{Acknowledgments}

The authors thank Hui Liao and Daniel Batista for their expert technical assistance with these studies.

This study was supported in part by the U.S. Public Health Service (R01 HL59488 and R01 HL55397), the American Heart Association (New York City Affiliate Grant-in-Aid, National American Heart Association Medical Student Research Fellowship to B.L. Hoh, and Clinician Scientist Award to D.J. Pinsky), the New York Academy of Medicine (Elsberg Fellowship to E.S. Connolly, Jr., and Glorney-Raisbeck Medical Student Award to B.L. Hoh), and the American Association of Neurological Surgeons (Young Clinician Investigator Award to E.S. Connolly, Jr.).

\section{References}

1. del Zoppo, G.J., B.R. Copeland, L.A. Harker, T.A. Waltz, J. Zyroff, S.R. Hanson, and E. Battenberg. 1986. Experimental acute thrombotic stroke in baboons. Stroke. 17:1254-1265.

2. del Zoppo, G.J. 1994. Microvascular changes during cerebral ischemia and reperfusion. Cerebrovasc. Brain Metab. Rev. 6:47-96.

3. Okada, Y., B. Copeland, R. Fitridge, J.A. Koziol, and G.J. del Zoppo.
1994. Fibrin contributes to microvascular obstructions and parenchymal changes during early focal cerebral ischemia and reperfusion. Stroke. 25:1847-1854.

4. Connolly, E.S., Jr., C.J. Winfree, D.M. Stern, R.A. Solomon, and D.J. Pinsky. 1996. Procedural and strain-related variables significantly affect outcome in a murine model of focal cerebral ischemia. Neurosurgery. 38:523-532.

5. Connolly, E.S., Jr., C.J. Winfree, T.A. Springer, Y. Naka, H. Liao, S.D Yan, D.M. Stern, R.A. Solomon, J-C. Gutierrez-Ramos, and D.J. Pinsky. 1996. Cerebral protection in homozygous null ICAM-1 mice after middle cerebral artery occlusion. Role of neutrophil adhesion in the pathogenesis of stroke. $J$. Clin. Invest. 97:209-216.

6. Connolly, E.S., Jr., C.J. Winfree, C.J. Prestigiacomo, S.C. Kim, T.F. Choudhri, B.L. Hoh, Y. Naka, R.A. Solomon, and D.J. Pinsky. 1997. Exacerbation of cerebral injury in mice which express the P-selectin gene: identification of P-selectin blockade as a new target for the treatment of stroke. Circ. Res. 81: 304-310.

7. Valles, J., M.T. Santos, A.J. Marcus, L.B. Safier, M.J. Broekman, N. Islam, H.L. Ullman, and J. Aznar. 1993. Downregulation of human platelet reactivity by neutrophils: participation of lipoxygenase derivatives and adhesive proteins. J. Clin. Invest. 92:1357-1365.

8. Lawson, C.A., S.-D. Yan, S.-F. Yan, H. Liao, G. Chen, J. Sobel, W. Kisiel, D.M. Stern, and D.J. Pinsky. 1997. Monocytes and tissue factor promote thrombosis in a murine model of oxygen deprivation. J. Clin. Invest. 99:17291738.

9. Ogawa, S., H. Gerlach, C. Esposito, A. Pasagian-Macaulay, J. Brett, and D.M. Stern. 1990. Hypoxia modulates the barrier and coagulant function of cultured bovine endothelium. J. Clin. Invest. 85:1090-1098.

10. Pinsky, D.J., M.C. Oz, S. Koga, Z. Taha, M.J. Broekman, A.J. Marcus, H. Liao, Y. Naka, J. Brett, P.J. Cannon, R. Nowygrod, T. Malinski, and D.M. Stern. 1994. Cardiac preservation is enhanced in a heterotopic rat transplant model by supplementing the nitric oxide pathway. J. Clin. Invest. 93:2291-2297.

11. Heye, N., C. Paetzold, R. Steinberg, and J. Cervos-Navarro. 1992. The topography of microthrombi in ischemic brain infarct. Acta Neurol. Scand. 86: 450-454.

12. Cortelazzo, S., G. Finazzi, M. Ruggeri, O. Vestri, M. Galli, F. Rodeghiero, and T. Barbui. 1995. Hydroxyurea for patients with essential thrombocythemia and a high risk of thrombosis. N. Engl. J. Med. 332:1132-1136.

13. Dietrich, W.D., S. Dewanjee, R. Prado, B.D. Watson, and M.K. Dewanjee. 1993. Transient platelet accumulation in the rat brain after common carotid artery thrombosis. An ${ }^{111}$ In-labeled platelet study. Stroke. 24:1534-1540.

14. Jafar, J.J., R. Menoni, H. Feinberg, G. LeBreton, and R.M. Crowell 1989. Selective platelet deposition during focal cerebral ischemia in cats. Stroke. 20:664-667.

15. Obrenovitch, T.P., and J.M. Hallenbeck. 1985. Platelet accumulation in regions of low blood flow during the postischemic period. Stroke. 16:224-234.

16. Kochanek, P.M., A.J. Dutka, K.K. Kumaroo, and J.M. Hallenbeck. 1988. Effects of prostacyclin, indomethacin, and heparin on cerebral blood flow and platelet adhesion after multifocal ischemia of canine brain. Stroke. 19:693-699.

17. Bronner, L.L., D.S. Kanter, and J.E. Manson. 1995. Primary prevention of stroke. N. Engl. J. Med. 333:1392-1400.

18. Gryglewski, R.J., S. Nowak, E. Kostka-Trabka, J. Kusmiderski, A. Dembinska-Kiec, K. Bieron, M. Basista, and B. Blaszczyk. 1983. Treatment of ischaemic stroke with prostacyclin. Stroke. 14:197-202.

19. Bederson, J.B., L.H. Pitts, and M. Tsuji. 1986. Rat middle cerebral artery occlusion: evaluation of the model and development of a neurologic examination. Stroke. 17:472-476.

20. Kottirsch, G., H.-G. Zerwes, N.S. Cook, and C. Tapparelli. 1997. $\beta$ amino acid derivatives as orally active non-peptide fibrinogen receptor antago- 
nists. Bioorg. Med. Chem. Lett. 7:727-732.

21. Bowie, E.J.W., and C.A. Owen, Jr. 1974. The bleeding time. In Progress in Hemostasis and Thrombosis. T.H. Spaet, editor. Grune and Stratton, New York. 249-271.

22. Naka, Y., N.C. Chowdhury, H. Liao, D.K. Roy, M.C. Oz, R.E. Michler, and D.J. Pinsky. 1995. Enhanced preservation of orthotopically transplanted rat lungs by nitroglycerin but not hydralazine. Requirement for graft vascular homeostasis beyond harvest vasodilation. Circ. Res. 76:900-906.

23. Mizutani, H., T. Furubayashi, A. Kuriu, H. Take, Y. Tomiyama, H. Yoshida, Y. Nakamura, M. Inaba, T. Yonezawa, S. Tarui, and S. Ikehara. 1990. Analyses of thrombocytopenia in idiopathic thrombocytopenic purpura-prone mice by platelet transfer experiments between (NZW X BXSB)F1 and normal mice. Blood. 75:1809-1812.

24. Lahiri, B., J.A. Koehn, R.E. Canfield, S. Birken, and J. Lewis. 1981. Development of an immunoassay for the $\mathrm{COOH}$-terminal region of the $\gamma$ chains if human fibrin. Thromb. Res. 23:103-112.

25. Emeis, J.J., J. Lindeman, and W. Nieuwenhuizen. 1981. Immunoenzyme histochemical localization of fibrin degradation products in tissues. Am. J. Pathol. 103:337-344.

26. Choudhri, T.F., B.L. Hoh, R.A. Solomon, E.S. Connolly, Jr., and D.J. Pinsky. 1997. Use of a spectrophotometric hemoglobin assay to objectively quantify intracerebral hemorrhage in mice. Stroke. 28:2296-2302.

27. Cook, N.S., H.G. Zerwes, C. Tapparelli, M. Powling, J. Singh, R. Metternich, and A. Hagenbach. 1993. Platelet aggregation and fibrinogen binding in human, rhesus monkey, guinea-pig, hamster and rat blood: activation by ADP and a thrombin receptor peptide and inhibition by glycoprotein IIb/IIIa antagonists. Thromb. Haemostasis. 70:531-539.

28. CAPRIE Steering Committee, 1997. A randomised, blinded, trial of clopidogrel versus aspirin in patients at risk of ischaemic events (CAPRIE). Lancet. 348:1329-1339.

29. Willette, R.N., C.F. Sauermelch, R. Rycyna, S. Sarkar, G.Z. Feuerstein, A.J. Nichols, and E.H. Ohlstein. 1992. Antithrombotic effects of a platelet fibrinogen receptor antagonist in a canine model of carotid artery thrombosis. Stroke. 23:703-711.

30. Coller, B.S., J.D. Folts, S.R. Smith, L.E. Scudder, and R. Jordan. 1989. Abolition of in vivo platelet thrombus formation in primates with monoclonal antibodies to the platelet GPIIb/IIIa receptor. Correlation with bleeding time, platelet aggregation, and blockade of GPIIb/IIIa receptors. Circulation. 80: $1766-1774$.

31. Zhang, Z., M. Chopp, R.L. Zhang, and A. Goussev. 1997. A mouse model of embolic focal cerebral ischemia. J. Cereb. Blood Flow Metab. 17:10811088 .

32. Winfree, C.J., C.J. Baker, E.S. Connolly, Jr., A.J. Fiore, and R.A. Solomon. 1996. Mild hypothermia reduces penumbral glutamate levels in the rat permanent focal cerebral ischemia model. Neurosurgery. 38:1216-1222.

33. Fisher, M., P.H. Levine, A.L. Fullerton, A. Forsberg, C.P. Duffy, J.J. Hoogasian, and D.A. Drachman. 1982. Marker proteins of platelet activation in patients with cerebrovascular disease. Arch. Neurol. 39:692-695.

34. Iwamoto, T., H. Kubo, and M. Takasaki. 1995. Platelet activation in the cerebral circulation in different subtypes of ischemic stroke and Binswanger's disease. Stroke. 26:52-56.

35. Kooten, F.V., G. Ciabattoni, C. Patrono, P.I.M. Schmitz, J.V. Gijn, and P.J. Koudstaal. 1994. Evidence for episodic platelet activation in acute ischemic stroke. Stroke. 25:278-281.

36. Weiss, H.J., J. Hawiger, Z.M. Ruggeri, V.T. Turitto, P. Thiagarajan, and T. Hoffman. 1989. Fibrinogen-independent platelet adhesion and thrombus formation on subendothelium mediated by glycoprotein IIb-IIIa complex at high shear rate. J. Clin. Invest. 83:288-297.

37. Hawiger, J. 1995. Mechanisms involved in platelet vessel wall interaction. Thromb. Haemostasis. 74:369-372.

38. Williams, M.J., X. Du, J. Loftus, and M.H. Ginsberg. 1995. Platelet adhesion receptors. Semin. Cell Biol. 6:305-314.

39. Parker, R.I., and H.R. Gralnick. 1986. Identification of platelet glycoprotein IIb/IIIa as the major binding site for released platelet von Willebrand factor. Blood. 68:732-736.

40. Robson, S.C., V.K. Young, N.S. Cook, G. Kottirsch, J.B. Siegel, B.A. Lesnokiski, D. Candinas, D.L.G. Wite, and F.H. Bach. 1996. Inhibition of platelet GPI IIb/IIIa in an ex vivo model of hyperacute xenograft rejection does not prolong cardiac survival. Xenotransplantation. 3:43-52.

41. Coller, B.S., K. Anderson, and H.F. Weisman. 1995. New antiplatelet agents: platelet GPIIb/IIIa antagonists. Thromb. Haemostasis. 74:302-308.

42. Willerson, J.T. 1996. Inhibitors of platelet glycoprotein IIb/IIIa receptors: will they be useful when given chronically? Circulation. 94:866-868.

43. Kotze, H.F., P.N. Badenhorst, S. Lamprecht, M. Meiring, V. Van Wyk, K. Nuyts, J.M. Stassen, J. Vermylen, and H. Deckmyn. 1995. Prolonged inhibition of acute arterial thrombosis by high dosing of a monoclonal anti-platelet glycoprotein IIb/IIIa antibody in a baboon model. Thromb. Haemostasis. 74: 751-757.

44. The EPIC Investigators, 1994. Use of a monoclonal antibody directed against the platelet glycoprotein IIb/IIIa receptor in high-risk coronary angioplasty. N. Engl. J. Med. 330:956-961.

45. Tcheng, J.E., A.M. Lincoff, K.N. Sigmon, M.M. Kitt, R.M. Califf, E.J. Topol, and The IMPACT II Investigators. 1995. Platelet glycoprotein IIb/IIIa inhibition with Integrilin during percutaneous coronary intervention: the IMPACT II Trial. Circulation. 92:540-543.

46. The RESTORE (Randomized Efficacy Study of Tirofiban for Outcomes and REstenosis) Investigators. 1997. Effects of platelet glycoprotein IIb/ IIIa blockade $\mathrm{E}$ with tirofiban on adverse cardiac events in patients with unstable angina or acute myocardial infarction undergoing coronary angioplasty. Circulation. 96:1445-1453.

47. The EPILOG Investigators. 1997. Platelet glycoprotein IIb/IIIa receptor blockade and low-dose heparin during percutaneous coronary revascularization. N. Engl. J. Med. 336:1689-1696.

48. Lincoff, A.M., and EPILOG Trial Group. 1996. Results of the interim analysis of the EPILOG trial. J. Am. Coll. Cardiol. 27:xxi.

49. Lind, S.E. 1991. The bleeding time does not predict surgical bleeding. Blood. 77:2547-2552. 